\title{
Instrumenting Smartphones with Portable NIRS
}

\section{Simon Klakegg}

Center for Ubiquitous Computing

University of Oulu

Oulu, Finland

simon.klakegg@ee.oulu.fi

\section{Chu Luo}

Center for Ubiquitous Computing

University of Oulu

Oulu, Finland

chu.luo@ee.oulu.fi

Jorge Goncalves

Center for Ubiquitous Computing

University of Oulu

Oulu, Finland

jorge.goncalves@ee.oulu.fi
Simo Hosio

Center for Ubiquitous Computing

University of Oulu

Oulu, Finland

simo.hosio@ee.oulu.fi

\section{Vassilis Kostakos}

Center for Ubiquitous Computing

University of Oulu

Oulu, Finland

vassilis@ee.oulu.fi

\begin{abstract}
In this paper we propose a mobile sensing solution that uses Near Infrared Spectroscopy (NIRS) and discuss its potential in future everyday use cases. The proposed design enables novice end users to classify various objects using NIRS and without prior knowledge of the technology itself. We describe how an instrument that traditionally has been used solely by trained lab personnel, can be commoditized to be used by any end user with a mobile device. The preliminary results indicate that samples can be identified with high accuracy, but that a series of implementation and design challenges must be first accounted for.
\end{abstract}

\section{Author Keywords}

Smartphones; ubiquitous computing; near infrared spectroscopy; mobile sensing.

\section{ACM Classification Keywords}

H.5.m. Information interfaces and presentation (e.g., $\mathrm{HCI}$ ): Miscellaneous;

\section{Introduction}

Smartphones have impacted many aspects of our lives over the recent years. The wide array of onboard sensors, constantly improving wireless connections and easy access to new applications have made these

devices integral parts of our everyday operations. One 
of the particularly interesting aspects of the modern smartphone, especially to researchers, is their ability to measure and sense the context around us (e.g., ambient light, temperature, etc.). This has effectively made the smartphone a capable scientific instrument as well. Given the ubiquity of smartphones, it is reasonable to say that these instruments are already at the hands of many users. We are interested in exploring a new generation of NIRS devices, and we argue that NIRS has the ability to turn smartphones into even more advanced analytical instruments for everyday use. NIRS utilizes the property between infrared light and molecular absorption to determine the physical properties of objects. It provides a range of benefits compared to traditional measuring methods used in industries, such as food, healthcare and pharmaceuticals. Recent breakthroughs in NIRS technology have facilitated making the sensing devices small, affordable and portable, while still retaining accuracy. Hence, we envision that smartphones can be paired with such devices, and thus allow users to benefit from advanced sensing capabilities. What used to be an expensive benchtop instrument in laboratories now has the possibility to reach numerous end users. To enable this transition, new design solutions should be developed to accommodate use by non-trained people. In this paper we first discuss the potential of this new technology, present the initial steps we have taken towards making the technology more accessible, and conclude by presenting a roadmap to tackle selected envisioned future challenges.

\section{Related Work}

Current smartphones are equipped with a wide array of built-in sensors (accelerometer, gyroscope, camera and GPS, etc.). These sensors can enhance user experience through advanced application features or help HCI researchers in collecting valuable data about the user's context $[1,9]$. Although using smartphones as scientific instruments is clearly feasible, the built-in sensors may not always be accurate enough for certain use cases. To this end, custom solutions can be developed and paired with the devices. For instance, an example of a custom sensing solution that can detect protein and allergens is explored by Long et al. [11]. Their setup allows the user to extract the physical parameters of a sample and achieve high precision fingerprinting. Identifying objects is a major research area in computer science [4]. A substantial body of work has been dedicated to pattern recognition and classifying objects. Gall et al. [8] propose a decision forest model to detect pedestrians or cars in natural images. In food science, an algorithm has been developed to recognize peel defects in citrus with up to $95 \%$ accuracy [3]. NIRS introduces an additional dimension to the analysis, being able to penetrate samples and expose information about its composition [14]. This has been proven useful in many industries, for example to detect concentrations of active substances in pharmaceutical tablets [7]. The technique has also demonstrated high accuracy in textile [5] and fruit quality classification [12].

\section{Portable NIRS}

Infrared (IR) spectroscopy was first introduced in the 1940s. It can be used to extract information about practically all types of samples, regardless of the state (e.g. liquids, solutions and powder). When an infrared signal $(780-2500 \mathrm{~nm})$ is radiated at a sample, it experiences absorption at various wavelengths

depending on the object's composition. The absorption is related to the vibration of the atoms in the molecules 
of the sample. Thus, detailed information about the inner configuration of the sample can be retrieved. The spectra of the resulting signal can be used for advanced analytics, allowing describing both physical and chemical parameters of the object being scanned. The technique is fast, accurate, non-destructive and requires little sample preparation. Traditionally, this technology has been reserved as an expensive laboratory instrument for trained personnel. Because of the extensive analytical capabilities of NIRS, researchers are working on developing portable solutions. An example is Texas Instrument's NIRS device, shown in Figure 1.

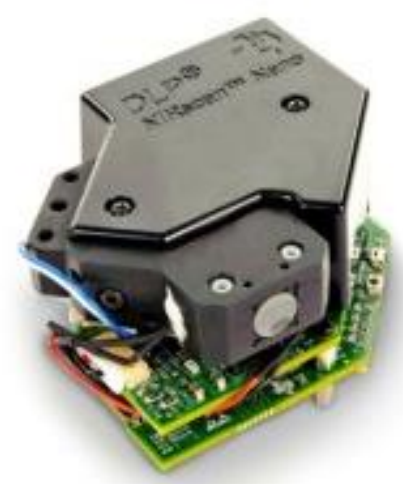

Figure 1. A Portable NIRS Device.

Due to the limited size and weight for mobile devices, there are some restrictions in the hardware. More specifically, there still exist minor differences in spectral resolution and sensitivity in contrast to traditional laboratory machines. Henn et al. [10] investigate how a miniaturized NIR spectroscopy performs compared to a benchtop device in quantitative food analysis. They report high accuracy for the portable NIRS, highlighting it as a viable alternative to conventional spectrometers, especially in non-critical use cases. With portable NIRS devices, one of the benefits is the ability to easily bring the analytical instrument to the sample. Normally, the sample would have to be collected and sent to a laboratory for processing. This is time consuming and requires significant effort due to the need of appropriate packaging, transportation and storage. Instead, the analysis using a portable device can now be performed in-situ, providing almost instantaneous results. This can be beneficial in many scenarios. For instance, picking grapes at the appropriate maturity level ensures that the grapes have all the enological conditions to become quality wine. Grape farmers could use the technology to determine the optimal time for harvest. A study on in-situ grape ripeness prediction [2] concludes with recommending a portable NIRS for the purpose of determining grape parameters. As the technology in spectrometers is based on comparing the results to a reference, a good library of scans will

increase the analytical value of the tool. Zamora-Rojas et al. [15] demonstrate how old scan libraries built by benchtop equipment can successfully be ported to new miniaturized scanners.

\section{Use Cases}

We envision a large number of potential application areas and use cases for a portable NIRS device paired with a mobile phone. We summarize these in Figure 2. For example, such a solution could aid authorities in identifying counterfeit or unclean pharmaceuticals. With an estimate of $7 \%$ of the sold drugs in the world being fake, there is certainly a need for efficient quality control. NIRS has already shown high accuracy in classifying tablets and antimalarial medicine [6]. Suspicious compounds could be scanned in-situ, giving 


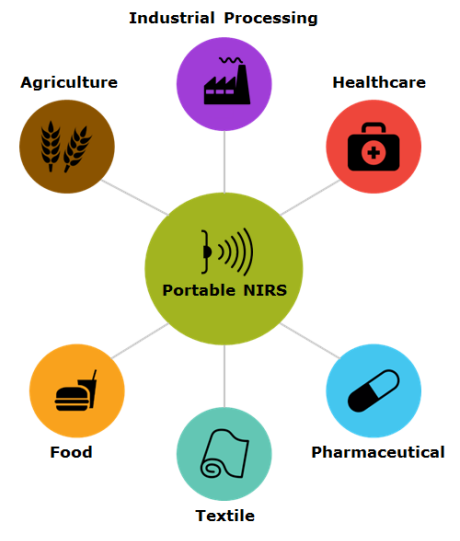

Figure 2: Potential use cases for NIRS. detailed analytical information in seconds. Similarly, another use case is to detect if meat has gone bad. The user can simply scan the meat and receive information about the quality and if it has decayed. NIRS can also be a useful tool for those with celiac disease, which is a frequent disorder in people with European origin. Gluten can be dangerous to those affected and it would be beneficial to have a quick, simple, effective and inexpensive method to detect gluten in food. NIRS can provide a solution, as it has been proven efficient in identifying gluten in ingredients such as flour.

\section{Implementation Challenges}

As NIRS primarily were utilized by trained personnel, device vendors have mainly focused on functionality, reliability and maintainability. To facilitate novice end users interacting with this technology, usability, efficiency and portability should be studied as well. Applications developed for such end user NIRS devices should provide users clear instructions on how to use the portable devices and provide understandable feedback based on the scan result. By designing and 3D printing a casing that encapsulates the NIRS device and informs the user about how to scan, the machine can act as a sensor in different areas of use. Larger scanning stations could be built, where the scanner is paired with public displays. These can then be placed in pivotal locations. This would allow people to simply walk in and scan their items (e.g., pills) in real time. In contrast, compact and visually appealing solutions are required to fit home environments such as kitchens. By placing NIRS in the hands of non-experts, it is also crucial to research the effect of different physical parameters on scanning reliability. Specifically, distance to the scanner, scanning angle, surface evenness, object interference, and stray light interference have all been shown to affect object identification accuracy in NIRS $[13,16]$. Figure 3 displays an overview of the challenges associated with NIRS and equipping novice end users with such an apparatus. When developing the physical casings and applications for portable NIRS, these metrics must be accounted for. The UI should instruct users on correct sample placement and scanning procedure. Samples holders must be designed to enable end users to position objects in an efficient and appropriate manner.

Challenges
Physical Parameters
Distance
Angle

Figure 3. Challenges associated with NIRS and end user involvement.

\section{Ongoing Work}

As smart devices (tablets, phones, watches) are becoming more and more available, we envision pairing them with NIRS devices to open up a significant number of exciting research avenues. In our already existing setup, the smart device acts as the interaction point, allowing users to configure settings, start scans and view the results near real-time. Figure 4 displays an overview of how user interaction of our approach compares to the traditional NIRS setup. Our users only interact with the smartphone to start the scans, and 
the results are presented to them by the smartphone client. The rest of the iterations, which previously required manual involvement, are automated by the application in our setup.

\begin{tabular}{|c|c|c|c|c|}
\hline & STEP & DESCRIPTION & $\begin{array}{l}\text { ORDINARY NIRS USER } \\
\text { INVOLVEMENT }\end{array}$ & $\begin{array}{l}\text { PROPOSED NIRS } \\
\text { USER INVOLVEMENT }\end{array}$ \\
\hline & Phone & UI Interaction & No & Yes \\
\hline ( د))))) & NIRS & $\mathrm{HW}$ Interaction & Yes & No \\
\hline n & Spectrum & Raw Data & Yes & № \\
\hline S & Analysis & $\begin{array}{l}\text { Filtering } \\
\text { Machine Learning }\end{array}$ & Yes & № \\
\hline (4) & Results & Interpretation & Yes & № \\
\hline & Phone & Presentation & No & Yes \\
\hline
\end{tabular}

Figure 4. An overview of how our design simplifies end user involvement.

As for concrete application scenarios, we are for example exploring how to leverage the apparatus for the elderly at their homes. Patients with memory problems or bad eyesight can be taught to verify their medicine using a custom-built NIRS deployment. The results can be presented either as text or transmitted by speech through a smart device. We have also started to collect samples of gluten in different types of popular food items, and expect to make a public library of the scan results. This could be helpful for people with celiac disease, as mentioned earlier. To further investigate the challenges associated with portable NIRS, we conducted several tests that verified the effects of physical parameters on scan results. We are currently exploring various 3D printed casing designs and mobile UI solutions, to mitigate these challenges in a real scanning situation with untrained end users. In addition to gluten traces, we have already built a library of various groups of items. We have achieved good preliminary results in classifying samples by using these reference scans in combination with preprocessing and machine learning algorithms.

\section{Conclusion}

This paper proposes a mobile instrumentation method, allowing novice smartphone end-users to perform advanced analysis of objects. We introduce a setup that pairs smart devices with a portable NIRS.

Implementing such a solution would open up new possibilities in ubiquitous smartphone sensing. Based on this system, we envision potential use cases and describe our ongoing work in the field. We discuss challenges when introducing novice end users to NIRS and how they can be solved through intuitive UI and design solutions. The purpose of this paper is to get early feedback and ideas from the mobile sensing community.

\section{Acknowledgements}

This work is partially funded by the Academy of Finland (Grants 276786-AWARE, 285062-iCYCLE, 286386CPDSS, 285459-iSCIENCE), and the European Commission (Grants PCIG11-GA-2012-322138, 645706-GRAGE, and 6AIKA-A71143-AKAI).

\section{References}

1. Ashiq Anjum and M. U. Ilyas. 2013. Activity recognition using smartphone sensors. In Consumer Communications and Networking Conference, IEEE, 914-919.

http://dx.doi.org/10.1109/CCNC.2013.6488584.

2. Federico E. Barnaba, Andrea Bellincontro and Fabio Mencarelli. 2014. Portable NIR-AOTF spectroscopy 
combined with winery FTIR spectroscopy for an easy, rapid, in-field monitoring of Sangiovese grape quality. Journal of the Science of Food and Agriculture 94, 6: 1071-1077.

3. J Blasco, N Aleixos and E Molto. 2007. Computer vision detection of peel defects in citrus by means of a region oriented segmentation algorithm. Journal of Food Engineering 81, 3: 535-543.

4. Chi-hau Chen, Louis-François Pau and Patrick S. -P. Wang. 2010. Handbook of pattern recognition and computer vision. World Scientific.

5. E Cleve, E Bach and E Schollmeyer. 2000. Using chemometric methods and NIR spectrophotometry in the textile industry. Analytica Chimica Acta 420, 2: 163-167.

6. Floyd E. Dowell, Elizabeth B. Maghirang, Facundo M. Fernandez, Paul N. Newton and Michael D. Green. 2008. Detecting counterfeit antimalarial tablets by near-infrared spectroscopy. Journal of pharmaceutical and biomedical analysis 48, 3: 1011-1014.

7. M Dyrby, S. B. Engelsen, L Nørgaard, M Bruhn and $L$ Lundsberg-Nielsen. 2002. Chemometric quantitation of the active substance (containing $\equiv$ $\mathrm{N}$ ) in a pharmaceutical tablet using near-infrared (NIR) transmittance and NIR FT-Raman spectra. Applied Spectroscopy 56, 5: 579-585.

8. Juergen Gall and Victor Lempitsky. 2013. Classspecific hough forests for object detection. Springer.

9. Manhyung Han, Young-Koo Lee and Sungyoung Lee. 2012. Comprehensive context recognizer based on multimodal sensors in a smartphone. Sensors 12, 9: 12588-12605.
10. Raphael Henn, Anita Schwab and Christian W Huck. 2016. Evaluation of benchtop versus portable near-infrared spectroscopic method combined with multivariate approaches for the fast and simultaneous quantitative analysis of main sugars in syrup formulations. Food Control 68: 97-104.

11. Kenneth D. Long, Hojeong Yu and Brian T. Cunningham. 2014. Smartphone instrument for portable enzyme-linked immunosorbent assays. Biomedical optics express 5, 11: 3792-3806.

12. Bart M. Nicolai, Katrien Beullens, Els Bobelyn, Ann Peirs, Wouter Saeys, Karen I. Theron and Jeroen Lammertyn. 2007. Nondestructive measurement of fruit and vegetable quality by means of NIR spectroscopy: A review. Postharvest Biology and Technology 46, 2: 99-118.

13. T. W. L. Scheeren, P Schober and L. A. Schwarte. 2012. Monitoring tissue oxygenation by near infrared spectroscopy (NIRS): background and current applications. Journal of clinical monitoring and computing 26, 4 : 279-287.

14. Barbara Stuart. 2005. Infrared spectroscopy. Wiley Online Library.

15. E Zamora-Rojas, D Pérez-Marín, E De Pedro-Sanz, J. E. Guerrero-Ginel and A Garrido-Varo. 2012 Handheld NIRS analysis for routine meat quality control: database transfer from at-line instruments. Chemometrics and Intelligent Laboratory Systems 114: $30-35$

16. M Zoccola, N Lu, R Mossotti, R Innocenti and $A$ Montarsolo. 2013. Identification of wool, cashmere, yak, and angora rabbit fibers and quantitative determination of wool and cashmere in blend: a near infrared spectroscopy study. Fibers and Polymers 14, 8: 1283-1289. 